\title{
Laser-Induced Breakdown Spectroscopy: Fundamentals, Applications, and Challenges
}

\author{
F. Anabitarte, A. Cobo, and J. M. Lopez-Higuera \\ Photonic Engineering Group, Department of TEISA, Universidad de Cantabria, Edificio I $+D+i$ Telecomunicacion, \\ 39005 Santander, Spain
}

Correspondence should be addressed to F. Anabitarte, anabitartef@unican.es

Received 12 September 2012; Accepted 1 October 2012

Academic Editors: H. J. Byrne and G. Louarn

Copyright (c) 2012 F. Anabitarte et al. This is an open access article distributed under the Creative Commons Attribution License, which permits unrestricted use, distribution, and reproduction in any medium, provided the original work is properly cited.

Laser-induced breakdown spectroscopy (LIBS) is a technique that provides an accurate in situ quantitative chemical analysis and, thanks to the developments in new spectral processing algorithms in the last decade, has achieved a promising performance as a quantitative chemical analyzer at the atomic level. These possibilities along with the fact that little or no sample preparation is necessary have expanded the application fields of LIBS. In this paper, we review the state of the art of this technique, its fundamentals, algorithms for quantitative analysis or sample classification, future challenges, and new application fields where LIBS can solve real problems.

\section{Introduction}

LIBS is an atomic emission spectroscopy technique which uses highly energetic laser pulses to provoke optical sample excitation [1]. The interaction between focused laser pulses and the sample creates plasma composed of ionized matter [2]. Plasma light emissions can provide "spectral signatures" of chemical composition of many different kinds of materials in solid, liquid, or gas state [3]. LIBS can provide an easy, fast, and in situ chemical analysis with a reasonable precision, detection limits, and cost. Additionally, as there is no need for sample preparation, it could be considered as a "put \& play" technique suitable for a wide range of applications [1]

Considerable progress has been made during the last few years on very different and versatile applications of LIBS, including remote material assessment in nuclear power stations, geological analysis in space exploration, diagnostics of archaeological objects, metal diffusion in solar cells, and so forth [4]. Today, LIBS is considered as an attractive and effective technique when a fast and whole chemical analysis at the atomic level is required.

Some of the established techniques for analytical atomic spectrometry are inductively coupled plasma-atomic emission spectrometry (ICP-AES), electrothermal atomizationatomic absorption spectrometry (ETA-AAS), and inductively coupled plasma-mass spectrometry (ICP-MS) [5], however, development on LIBS during recent years has reduced its gap in performance with respect to these other well-known approaches [5].

This paper begins with a brief explanation of the physics involved in plasma induction and the features of this plasma in LIBS and is then followed by a description of the basic devices which compose a LIBS set-up. These devices will be described associating their features with the properties of the induced plasma. Moreover, different kinds of analysis algorithms will be introduced in order to go beyond the "spectral signatures" obtained with the technique. Finally, some key LIBS applications will be described, and the main research challenges that this approach faces at the moment will be discussed.

\section{Fundamentals of Plasma Physics and Its Spectra}

Understanding the plasma physics of LIBS is essential to provide an optimized setting for LIBS measurements. A large number of environmental factors affect the plasma life time and features, changing the spectral emission and the 
performance of this technique for chemical analysis at the atomic level.

\subsection{Laser Ablation and Plasma Physics of LIBS. Laser-} matter interactions are governed by quantum mechanics laws describing how photons area absorbed or emitted by atoms. If an electron absorbs a photon, the electron reaches a higher energy quantum mechanical state. Electrons tend to the lower possible energy levels, and in the decay process the electron emits a photon (deexcitation of the atom). The different energy levels of each kind of atom induces different and concrete photon energies for each kind of atom, with narrowband emissions due to the quantization, with an uncertainty defined by Heisenberg uncertainty principle. These emissions are the spectral emission lines [6] found in LIBS spectra and its features and their associated energy levels are well known for each atom [7].

If the energy applied to the atom is high enough (overcoming the ionization potential), electrons can be detached by the atom inducing free electrons and positive ions (cations). Initially, the detached electron is the most external one (the furthest with respect to the nucleus) because it has the lowest ionization potential, but with higher energy supply it is possible to detached more electrons overcoming the second ionization potential, the third, and so on. These ions can emit photons in the recombination process (cations absorbs a free electron in a process called free-bound transition) or in the deexcitation process (the cations and the electrons lose energy due to kinetic process in a process called free-free transition). These emissions can be continuum due to the different energies of the ions and the different energy transitions, however cations deexcitation has discrete (or quantized) set of energy levels with characteristic emission lines for each kind of element, allowing its identification together with the atomic emission lines [7].

The plasma, induced by the interaction pulsed lasersample, emits light which consists of discrete lines, bands, and an overlying continuum. These discrete lines, which characterize the material, have three main features; wavelength, intensity, and shape. These parameters depend on both the structure of the emitting atoms and their environment. Each kind of atom has some different energy levels which determine the wavelength of the line. Besides the identification of the elements in the sample, the calculation of the amount of each element in the sample from the line intensities is possible taking in account different necessary conditions fixed by local thermodynamic equilibrium (LTE condition) or problems related with matrix effects which can reduce the accuracy of quantitative analysis $[8,9]$.

On the other hand, the intensity and shape of the lines depend strongly on the environment of the emitting atom. For not too high plasma densities, both the natural broadening (due to Heisenberg's uncertain principle) and the Doppler broadening (Doppler Broadening is due to the thermal motion of the emitters, the light emitted by each particle can be slightly red- or blue-shifted, and the final effect is a broadening of the line) dominate the linear shape [10]. For high plasma densities, atoms in the plasma are affected by electric fields due to fast moving electrons and slow moving ions, and these electric fields split and shift the atomic energy levels. As a consequence of these perturbations of the levels, the emission lines are broadened and they change their intensity and shape. This effect is known as the Stark effect [10] and it dominates the line shape for dense plasmas. This broadening together with the different parameters of spectral lines (intensities and shapes) and even the continuum radiation features can be useful to determine plasma parameters, such as electron temperature, pressure, and electron density $[2,7]$. These parameters are very important to characterize the plasma, giving information about the physical state of it. Moreover the calculation of these parameters is necessary because the set-up has to be tuned to ensure LTE, key condition for an accurate quantitative analysis [11].

Basically, there are three stages in the plasma life time (Figure 1). The first one is the ignition process. This process includes bond breaking and plasma shielding during the laser pulse, depending on laser type, irradiance, and pulse duration.

If the selected laser is a femtosecond one, nonthermal processes will dominate the ionization. The pulse is too short to induce thermal effects; hence other effects should ionize the atoms, depending of the kind of sample. The pulse has a huge amount of energy and effects like multiphoton absorption and ionization, tunneling, and avalanche ionization excite the sample. With this amount of energy, the electron-hole created will induce emission of X-rays, hot electrons, and photoemission. This will create highly charged ions through a process called Coulomb explosion [7]. The absence of thermal effects creates a crater with highly defined edges without melted or deposited materials.

In contrast, nanosecond lasers induce other effects. The electron-lattice heating time is around $10^{-12} \mathrm{~s}$, much shorter than the pulse time. This causes thermal effects to dominate the ionization process. Briefly, the laser energy melts and vaporizes the sample, and the temperature increase ionizes the atoms. If the irradiance is high enough, nonthermal effects will be induced too and both will ionize the sample. Between $10^{-9} \mathrm{~s}$ and $10^{-8} \mathrm{~s}$, plasma becomes opaque for laser radiation, thus the last part of the laser pulse interacts with plasma surface and will be absorbed or reflected, hence it will not ionize much more material. This effect is called plasma shielding and is strongly dependent on environmental conditions (surrounding gases or vacuum) and experimental conditions (laser irradiance and wavelength) $[12,13]$. This shielding reduces the ablation rate because the radiation does not reach the sample surface. This induces a crater with melted and deposited material around it but at the same time the plasma is reheated and the lifetime and size of plasma is higher $[13,14]$.

The next step in plasma life is critical for optimization of LIBS spectral acquisition because the plasma causes atomic emission during the cooling process. After ignition, the plasma will continue expanding and cooling. At the same time, the electron temperature and density will change. This process depends on ablated mass, spot size, energy coupled 
to the sample, and environmental conditions (state of the sample, pressure, etc.).

If the plasma is induced in a vacuum, the plasmaplume expands adiabatically and the expansion of the ablated material can be described by the Euler equations of hydrodynamics [15]. In contrast, if the surrounding medium is a gas or a liquid, the plume will compress the surrounding medium and produce shockwaves. In this situation the plasma plume is a mixture of atoms and ions from both vaporized material and ambient gas. The shockwave expansion can be described by Sedov's theory $[7,16]$.

Plasma temporal evolution changes with pulse duration. For pulses longer than about 5ps, the laser-plasma interactions result in plasma heating and the plasma temperature increases with pulse duration [17]. For short times (below $30 \mathrm{~ns}$ ) a fs-induced plasma emission intensity decays while ns-induced plasma becomes hotter [18]. The last part of the nanosecond laser pulse is absorbed by the plasma, reheating it, elongating lifetime, and increasing line emission, but at the same time, the background is higher and this decreases the sensitivity for nanosecond set-ups.

A good way to improve the performance is to use a femtosecond laser to ablate the sample and nanosecond or picosecond pulses to reheat the plasma [19].

The last stage of the plasma life is not interesting for LIBS measurements. A quantity of ablated mass is not excited as vapor or plasma, hence this amount of material is ablated as particles and these particles create condensed vapor, liquid sample ejection, and solid sample exfoliation, which do not emit radiation. Moreover, ablated atoms become cold and create nanoparticles in the recombination process of plasma.

2.1.1. Plasma Emission Spectra. The emission lines from the atomic species can be hidden by continuum radiation that is caused by two processes. The first one is due to radiative recombination. Both continuum and line photons can be produced in such a recombination event as the electron passes from the free state into the upper bound levels of the ion and then cascades down to form a ground state ion. The other effect involved in continuum radiation is called Bremsstrahlung. This effect is related to free-free transitions corresponding to the loss of kinetic energy by an electron in the field of an ion. The electron loses energy in the deceleration process when it travels into the field of the ion, emitting photons in different wavelengths, depending of its initial energy and the loss of this energy. Continuum emission can hide atomic emission peaks, thus this effect should be avoided.

The continuum emission depends on both temperature and density of plasma. These parameters are too high in the initial stages of plasma, especially in the ignition. For this reason, the time control unit has to delay the acquisition window in order to avoid this continuum. For femtosecond lasers, the continuum emission is observed within one nanosecond after the laser ablation; hence the delay with these lasers should be greater than this time. For nanosecond lasers, atomic emission occurs after 1

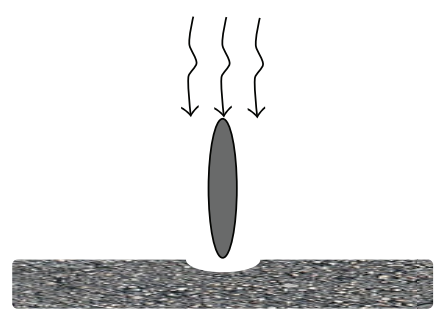

(a)

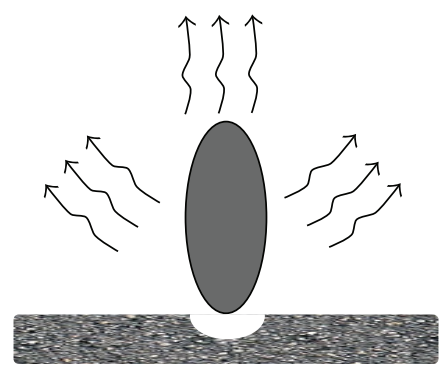

(b)

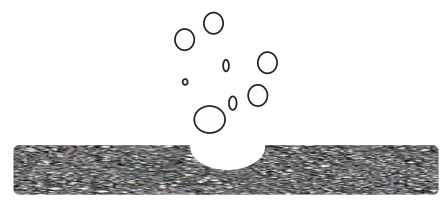

(c)

Figure 1: Plasma life stages: (a) plasma ignition, (b) plasma expansion and cooling, and (c) particle ejection and condensation [7].

microsecond and molecular emission occurs at later times from recombination of species in the plasma $[2,7,14]$. Inside this acquisition window, the initial stages of plasma life are characterized by a higher temperature and electron density. These parameters provide a better emission of ionic lines, for that reason these initial stages are better to acquire ionic lines despite the continuum emission. These optimal acquisition windows depend strongly on the sample material and both environmental and experimental conditions, but the values given above can be interesting as a starting point for each kind of laser [20].

Lifetime for femtosecond-induced plasmas is shorter and with less background. For that reason, the acquisition window size should be shorter for short pulses and femtosecond lasers are better for nongated measurements because the background is weak and the LIBS sensitivity improves [14].

Generally, femtosecond lasers are better to obtain highly accurate craters and for nongated measurements, and using delays of a few nanoseconds and small acquisition windows can improve LIBS sensitivity. Nanosecond lasers create a melted crater and need delays of a few microseconds and larger windows with the advantages of low complexity in the laser system. There are other kinds of lasers, such as picosecond lasers, for which the time pulse is between a femtosecond and a nanosecond, hence the features are between the two [7]. 


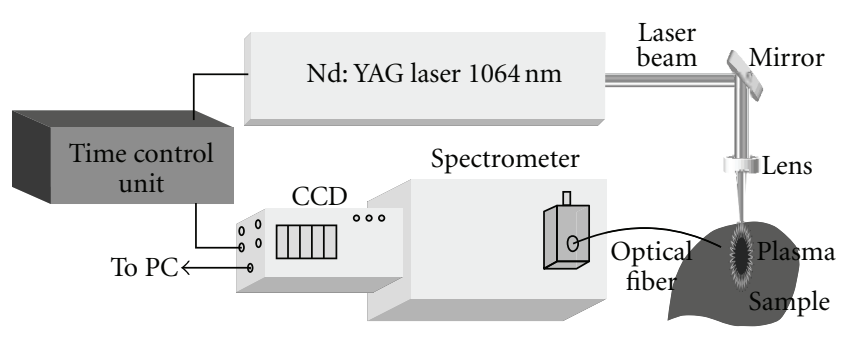

FIGURE 2: Typical LIBS set-up.

2.2. Environment. The plasma size, propagation speed, energy, and emission properties are related to the ambient gas into which the plasma expands. The ambient gas can help or prevents the plasma shielding. For example, the gas can shield the sample from the laser beam if a gas breakdown occurs before sample vaporization [21]. These undesirable effects are less important for gases and aerosols, but they can be important for solid samples.

Gas pressure will influence plasma expansion. Low pressures increase energy losses and uniformity of the plasma energy distribution. In addition, different gases have different behaviors at different pressures [21].

The laser can be directed anywhere, allowing the researcher to work near to the sample or far from it, for instance with dangerous samples like explosives [22] in Stand-off configurations. A Stand-Off LIBS (ST-LIBS) is defined as a LIBS set-up which allows a remote, noncontact material detection-characterization over a distance of at least several meters [23]. This feature of LIBS enables flexible setups adapted to dangerous environments or samples.

\section{LIBS Set-Up Components}

The main devices involved in a LIBS analysis are shown in Figure 2. A high-energy pulsed laser (usually in the nanosecond range) is directed at the sample [2]. This light energy vaporizes the sample and induces the plasma.

The spectrometer is in charge of diffracting the light collected, with a more or less complex optical system, in order to obtain the spectral signature. Then, the light is detected by using devices such as a photomultiplier tube (PMT), a photodiode array (PDA), or a charge-coupled device (CCD) [2, 7]. Finally, the acquired spectrum is processed by a computer for further analysis. LIBS set-ups need an accurate time control to avoid some plasma life stages and to improve the spectral signature $[2,3,7]$. The choice of the laser combined with the set spectrometer-detector and time control, adapted to environmental conditions, can determine the success or failure of the experiment.

3.1. Laser Configurations for LIBS. The main device of LIBS is the laser. It generates the energy to induce the plasma and mainly determines the plasma features. The main parameters related to the laser are the pulse time (explained above), the energy per pulse, the wavelength, and the number of pulses per burst [24]. Obviously, each application works better with a combination of these parameters. Nanosecond-pulsed lasers are the most common for LIBS. Therefore, the most of this section is related to this kind of laser.

3.1.1. Laser Wavelength. The wavelength influence on LIBS can be explained from two points of view; the laser-material interaction (energy absorption) and the plasma development and properties (plasma-material interaction).

When photon energy is higher than bond energy, photon ionization occurs and nonthermal effects are more important. For this reason, the plasma behavior depends on wavelength in nanosecond LIBS set-ups. In the same way, the optical penetration is shorter for UV lasers, providing higher laser energy per volume unit of material. In general, the shorter the laser wavelength, the higher the ablation rate and the lower the elemental fractionation [25].

The plasma ignition and its properties depend of wavelength. The plasma initiation with nanosecond lasers is provoked by two processes; the first one is inverse Bremsstrahlung by which free electrons gain energy from the laser during collisions among atoms and ions. The second one is photoionization of excited species and excitation of ground atoms with high energies. Laser coupling is better with shorter wavelengths, but at the same time the threshold for plasma formation is higher. This is because inverse Bremsstrahlung is more favorable for IR wavelengths [26].

In contrast, for short wavelengths (between 266 and $157 \mathrm{~nm}$ ) the photoionization mechanism is more important. For this reason, the shorter the wavelength in this range, the lower the fluence necessary (energy per unit area) to initiate ablation [27]. In addition, when inverse Bremsstrahlung occurs, part of the nanosecond laser beam reheats the plasma. This increases the plasma lifetime and intensity but also increases the background at the same time. Longer wavelengths increase inverse Bremsstrahlung plasma shielding, but reduce the ablation rate and increase elemental fractionation (elemental fractionation is the redistribution of elements between solid and liquid phases which modifies plasma emission) [28].

The most common laser used in LIBS is pulsed Nd:YAG [29]. This kind of laser provides a compact, reliable, and easy way to produce plasmas in LIBS experiments. The fundamental mode of this laser is at $1064 \mathrm{~nm}$ and the pulse width is between 6 and 15 ns. This laser can provide harmonics at 532, 355, and $266 \mathrm{~nm}$, which are less powerful and have shorter time pulses (between 4 and $8 \mathrm{~nm}$ ) [2, 7]. The fundamental and the first harmonic are the most common wavelengths used in LIBS. This harmonics can be useful to work with different wavelengths in the same environmental conditions, because a lot of Nd:YAG lasers can produce all of them. Other kinds of lasers can be used in LIBS, such as $\mathrm{CO}_{2}$ or excimer lasers to work in far IR or UV ranges, respectively. Lasers based on fiber or dye technology can reduce the pulse width if the user is attempting to work with picosecond or femtosecond pulses.

3.1.2. Laser Energy. The energy parameters related with laser material interaction are fluence (energy per unit area, $\mathrm{J} / \mathrm{cm}^{2}$ ) 
and irradiance (energy per unit area and time, $\mathrm{W} / \mathrm{cm}^{2}$ ). Ablation processes (melting, sublimation, erosion, explosion, etc.) have different fluence thresholds [30]. The effect of changes in the laser energy is related to laser wavelength and pulse time. Hence, it is difficult to analyze the energy effect alone. In general, the ablated mass and the ablation rate increase with laser energy.

The typical threshold level for gases is around $10^{11} \mathrm{~W} / \mathrm{cm}^{2}$ and $10^{10} \mathrm{~W} / \mathrm{cm}^{2}$ for liquids, solids, and aerosols [2]. These values are for guidance and depend strongly on laser pulse time and environmental conditions, reaching up to around $10^{15} \mathrm{~W} / \mathrm{cm}^{2}$ for nitrogen at 760 Torr with a Nd:YAG laser, $1064 \mathrm{~nm}, 7 \mathrm{ps}$ [2].

3.1.3. Acquisition Time and Delay. The first stages of LIBSinduced plasma are dominated by the continuum emission. The time gate of decay of this continuum radiation change with a wide range of experimental parameters, such as laser wavelength and pulse time, ambient pressure or sample features. Besides, these experimental parameters fixes set the time periods of atomic emission, the most interesting stage of LIBS plasmas.

Plasma evolution using an IR (Nd:YAG) and a UV (excimer) lasers has been analyzed to discover differences induced by laser wavelength [31]. The plasma continuum emission stage was around $400 \mathrm{~ns}$ for UV laser and several microseconds for IR laser. Laser wavelength can affect the selection of delay time (gate delay) and integration time (gate window) and these parameters are essential to optimize the signal to background ratio. The analysis of plasma evolution for $\mathrm{Zn}$ and $\mathrm{Cd}$ in sand has been analyzed in other works, with an optimal gate delay of $0.5 \mu \mathrm{s}$ and a gate window of $1.5 \mu \mathrm{s}$ [32].

This analysis of optimal gate delay and window can be achieved optimizing the signal to background ratio and the repeatability of this parameter. For a Nd:YAG laser at the fundamental wavelength with power density of approximately $2 \mathrm{GW} / \mathrm{cm}^{2}$, the best compromise between lower relative standard deviation (R.S.D.) and higher signalto-background ratio was found at a delay of approximately $6 \mu \mathrm{s}$. The integration time was fixed at $15 \mu \mathrm{s}$ [20].

There are different points of view to optimize the gate delay and window, and the big amount of experimental parameters involved in plasma evolution makes it difficult to recommend valid values for these parameters. The selection should be determined case by case in order to achieve a compromise between high-line intensity and low background. Briefly, for Nd:YAG lasers, both times for gate delay and gate window are in the order of microseconds but this values can change if another kind of results are sought.

3.1.4. Sequences of Pulses: Double Pulse LIBS. Proposed twenty-eight years ago to improve the detection limits of some elements [33], dual pulse LIBS configuration consists on the sequence of two laser pulses temporally spaced in the order of few nanoseconds or microseconds (depending on laser pulse time, the larger the pulse time, and the larger the time delay between pulses). These two pulses can excite the

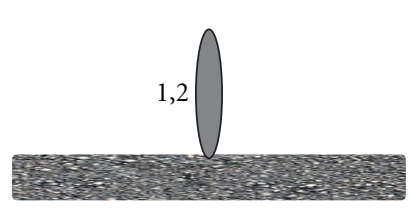

(a)

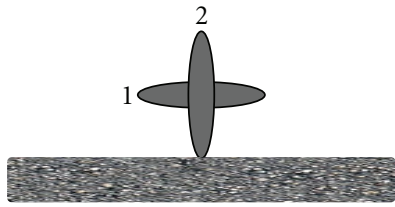

(b)

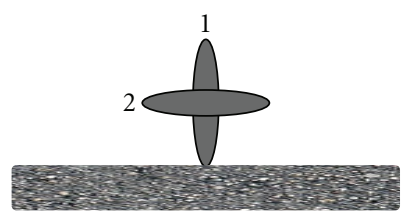

(c)

FIgURE 3: Dual pulse configurations (a) shows collinear configuration; the first pulse ablates the sample and the second one reheats the plasma [34], (b) is an orthogonal pre-ablative configuration; the first pulse creates a spark on the surrounding media and the second one ablates the sample $[14,35]$, (c) shows the same idea as (a), but the plasma is reheated in an orthogonal way [36].

same area and create two temporally spaced plasmas or the second pulse can reheat the plasma induced by the first one.

Using dual pulse LIBS, atomic emission, signal-to-noise ratio, and limit detection can be improved. Conversely, dual pulse LIBS can complicate the LIBS set-up, although the benefits can justify this complication. These improvements provide better detection limits than single pulse configurations due to the induced atomic emission enhancement. Figure 3 shows the most common dual pulse configurations.

The signal emission enhancement is due to different factors in these dual pulse configurations. This enhancement in collinear and orthogonal reheating is due to energetic issues. In collinear configurations, the second pulse increases the ablated material and, mainly, reheats the plasma, increasing its volume and its emission, but if the time between laser pulses is enough to allow the fading of the plasma, the second pulse interacts with the sample and ablates more material for the reduced atmosphere induced by the first pulse. In the orthogonal configuration, the second pulse does not ablate more material, but reheats the plasma and reexcites the material ablated by the first pulse. Other authors have given reasons for the signal enhancement on preheating orthogonal configurations, such as a reduction in density or pressure of the surrounding media due to the first pulse $[35,37-39]$.

3.2. Spectrometers and Detectors. The spectrometer or spectrograph is a device which diffracts the light emitted by the plasma. There are different designs, such as Littrow, Paschen-Runge, Echelle, and Czerny-Turner [40, 41]. The Czerny-Turner spectrograph is the most common device in LIBS. This spectrograph is composed of an entrance slit, two mirrors, and a diffraction grating. The light comes through the slit and reaches the first mirror which collimates the light, directing it onto the grating. Light is reflected at different angles according to its wavelength. The second 


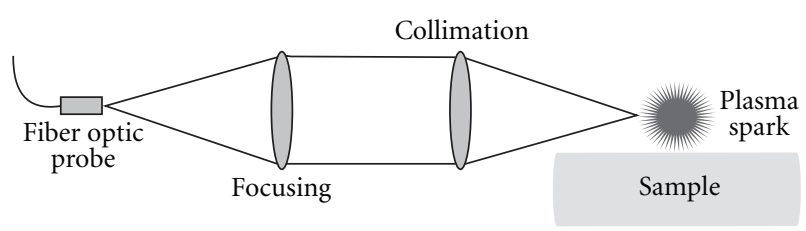

Figure 4: Typical light acquisition system.

mirror focuses the light on the focal plane where the detector is placed.

In recent years, the Echelle spectrograph has been used more extensively [41]. The Echelle spectrograph uses a diffraction grating placed at a high angle, producing a large dispersion in a small wavelength range in each order. As the orders are spatially mixed, a prism is used to separate them. The orders are stacked vertically on the focal plane. For that reason, Echelle devices need a two-dimensional detector. Each vertical portion of the detector contains a part of the spectra and the software composes the whole spectrum.

Different kinds of detectors are used in LIBS, depending of the application. To measure light intensity without spectral decomposition, the photomultiplier tube (PMT) or avalanche photodiode (APD) can be used. On the other hand, for one-dimensional spatial information, the researcher can combine a spectrograph and a photodiode array (PDA) or an intensified photodiode array (IPDA) for time-resolved measurements.

If two-dimensional spatial information is required, the most common devices are charge coupled devices (CCD) and intensified CCD (ICCD). A CCD detector provides less background signal, although ICCD improves the signal-tonoise ratio and is better for time-resolved detection using windows of a few nanoseconds [42]. Another problem related to ICCDs is the price, which is much higher than CCDs.

3.3. Collecting and Focusing Light. There are two main parts related to light acquisition and focusing. Typical laser spots currently must be focused to increase the irradiance to induce plasma formation. Light from plasma must then be collected using devices such as optical fibers, lenses, or combinations of both in order to guide it to the spectrometer.

3.3.1. Focusing Laser Pulses with Lenses. As the laser beam width from the majority of solid-state lasers is of the order of 6-8 $\mathrm{mm}$, the most common lenses used to focus laser pulses have diameters of 25 or $50 \mathrm{~mm}$ and focal lengths between 50 and $150 \mathrm{~mm}$. There are other applications such as standoff set-ups where a multilens system or different lenses are required. The material of the lens should have maximum transmission at laser wavelength and they must be coated with antireflection layers to minimize transmission losses (the laser beam can lose around $8 \%$ of its energy passing through an uncoated lens. Antireflection coatings can reduce this ratio to around $0.5 \%$ ).
3.3.2. Collecting Light. A common set-up to acquire plasma light is shown in Figure 4. The first lens collimates the emitted light to improve the focalization of the second one into the fiber probe and to optimize the ratio of acquired light. This set-up can be adapted to different systems using only one lens, a multilens device, and even only the fiber probe positioned in front of the spark. The features of lenses used to focus the plasma light are useful for this purpose, adapting them to different environmental features or set-ups.

3.3.3. Optical Fibers. Sometimes, the sample is far from the detection system, from the lasers, or from both of them, and so a system based on lenses like the one shown in Figure 4 is unpractical [43]. In these situations or when the environment is too aggressive or the access restriction makes it difficult to induce the plasma or acquire the light, fiber optic cables (FOCs) or bundles are used [44-46]. Although the most common optical fibers in LIBS are made of fused silica (with diameters between $50 \mu \mathrm{m}$ and $1 \mathrm{~mm}$ ), different kinds of fibers such as photonic-crystal fibers are used too [47]. Approaches using these coiled fibers (including plastic optical fiber (POF) ones) placed around the plasma plume are also used $[48,49]$.

Optical fiber technologies can be used to detect other significant signals from plasma such as shockwaves [46]. Plasma compresses the surrounding media and generates a shockwave. This shockwave can be detected with classical microphones [50, 51]. However, shockwaves can also be detected using other devices based on optical fiber such as Fiber Bragg Grating [52]. In addition, with this technology based on periodical refractive index changes in the fiber core, temperature, or strain, among other factors, can be detected and measured $[53,54]$.

\section{Algorithms for Quantitative and Qualitative Analysis}

The main goal of LIBS is to achieve a chemical analysis at the atomic level, either qualitatively (i.e., to assess the presence of particular elements) or quantitatively, in which the relative amount of different elements in the sample is quantified from the processing of the acquired spectra. In both cases, a key step is the proper identification of each emission line of a particular element in a neutral or ionized state [2]. If the sample composition is known approximately, the setup can be adapted to find the optimal spectral range where there are emission lines of the elements under analysis or to discard emission lines of elements outside the sample. At the same time, it is interesting to know the expected relative intensity of each line. If there are doubts about one line that could belong to different elements with very close emission wavelengths, the most probable element can be inferred from the relative intensity and other theoretical and empirical information about emission probabilities [55]. By the same token, the ionization stage is important too. If two elements in different ionization stages are possible for the same emission line, the element in neutral stage should be more likely. Even more, experimental conditions 
can determine the emission lines in the spectral signature. For example, lines from $\mathrm{Fe}(\mathrm{I})$ and $\mathrm{Fe}(\mathrm{II})$ are possible in air, but in vacuum, $\mathrm{Fe}(\mathrm{III})$ is possible too because the ionization potential of second ionization stage is lower in this medium.

The latest research in LIBS has tried to improve the accuracy of quantitative chemical analysis. Quantitative analysis has to provide the concentration of species in the sample (parts per million), the absolute mass of species (ng in a particle), or a surface concentration $\left(\mathrm{ng} / \mathrm{cm}^{2}\right)$. This analysis usually finishes in a calibration curve, but this curve is strongly dependent on experimental conditions. For this reason, the conditions should be the same when a new sample is analyzed, and sometimes this is difficult because there are many factors that can change them. An averaging of many acquired spectra can attenuate the variation of spectral emission for the analysis, but the pulse-to-pulse variation can be too high to attempt quantitative analysis.

These factors involve aspects both from LIBS set-up and environmental and sample conditions. Laser pulse energy, repetition rate, and detector gain and linearity, as well as lens-to-sample distance (LTSD) can render useless the calibration curve [56, 57]. Calibration curves can be affected by environmental conditions like changes in the atmosphere around the sample or in the light path [58]. At the same time, uniformity in sample composition, surface roughness, or matrix effects affect the accuracy [59]. All these effects induce the variation of the emission intensity between spectra of the same sample.

Matrix effects, that is, the changes in the emission intensity of some elements when the physical properties or composition of the sample varies, greatly affect the accuracy of quantitative estimations in LIBS. For instance, the emission intensity of silicon in water or in soil changes, even if the concentration in both samples is the same. There are two kinds of matrix effects. The first one is called physical matrix effect and is related with the fact that physical properties of the sample can change the ablation parameters. These changes alter the amount of ablated mass; hence the emission intensity changes although the concentration is the same in different matrices. Chemical matrix effects, on the other hand, occur when the emission features of one element are altered by the presence of another one.

One common solution is to use calibration curves, obtained from materials of known composition. A calibration curve is a method for determining the concentration of a substance in an unknown sample by comparing the unknown to a set of standard samples of known concentration. The result is a curve composed by values for the desired quantity as a function of values of sensor output (in LIBS, emission peaks of the element under analysis obtained from LIBS spectra). The curve is created with the measurements of known concentrations and an unknown concentration of a new sample can be predicted using the measurement obtained using its LIBS spectrum. However, many calibrated samples within the expected range of composition variation are required. Another technique, internal standardization, is based on a particular element whose concentration in the sample is constant (it can be added to the sample if needed), and its measurement is used to correct the response to other elements [60]. On the other hand, the impact of other effects (such as unwanted changes in shot-to-shot laser pulse parameters) on the accuracy of the quantitative analysis can be reduced by the so-called external standardization. In this case, an indirect physical magnitude is used to correct (i.e., to normalize) the spectra. For example, it has been observed that during plasma expansion, a shockwave is induced in the surrounding media, and the energy of this shockwave is related to the plasma energy and can be used to reduce the unwanted variations of the spectra $[51,61]$. This shockwave can be acquired with a microphone [51] or with other kinds of detectors, such as optical fiber pressure sensors [54].

Furthermore, there is a technique that solves the problem related to the calibration of LIBS, called calibration-free LIBS $[9,62]$. This procedure applies an algorithm in order to avoid matrix effects and the use of calibration curves, providing a fast and accurate quantitative analysis. This algorithm assumes that plasma is LTE, mandatory condition for all kind of quantitative analysis using LIBS, the radiation source is thin and the plasma composition represents the sample composition, typical conditions for LIBS plasmas. The algorithm calculates the line integral intensity between two energy levels of an atomic species, and using different lines at different energy levels, it can calculate the concentration of each element in the sample without calibration from these lines' integral intensities. Theoretically, this algorithm can calculate the concentration of all the elements in the sample up to the detection limit of the method [9].

4.1. Sample Classification Algorithms. The capability of LIBS to detect atomic emission of all chemical elements enables an accurate qualitative chemical analysis. However, there are applications where this kind of analysis is not necessary, for example, if two different materials need to be distinguished and classified. In this case, the raw spectra provided by LIBS can be processed by classification algorithms for distinguishing between different samples.

Algorithms such as artificial neural networks (ANN) $[63,64]$, support vector machines (SVM) $[64,65]$, or Knearest neighbors $(\mathrm{Knn})[66,67]$ belong to this group. These algorithms need a large training dataset to obtain an accurate classifier and need time to train (SVM and ANN) or need time to classify but not to train (Knn), but the results are usually excellent. These algorithms and other ones can provide a "chemometric" analysis (Powerful statistical signal-processing techniques which provide the automatic identification of chemical information, like spectral fingerprints).

An important aspect of the spectral data provided by LIBS and many other spectroscopic methods is that each spectrum contains a lot of redundant information. In LIBS, in particular, only a few emission lines at particular wavelengths may be interesting to perform the required classification. Redundancy can reduce the accuracy of classifiers and sometimes needs to be avoided. In addition, classification or training time is reduced if the input dataset has less information. 
There are two different kinds of algorithms that discard redundant information. The first group is composed of algorithms which select the most discriminating features. These algorithms, such as Sequential Floating Forward Selection (SFFS) [68] or Linear Discriminant Analysis (LDA) [69] select the best features of the input dataset in order to improve the classification ratio (in LIBS, the best wavelengths). The second group includes algorithms which extract features, such as principal component analysis (PCA) [70]. These algorithms combine the features of the input dataset and generate new features. Both groups, conveniently adjusted, improve the capability of classifiers.

These algorithms and other ones such as supervised learning algorithms or genetic algorithms have recently been applied to LIBS [71-73]. These works analyze samples ranging from metallic alloys, soils, heavy metals in water to toxins in toys, and can classify them or detect traces of a particular element, automatically.

A great research effort is being devoted to going beyond a mere spectra-capturing device. With the powerful algorithms described above, an integrated and portable device based on LIBS that can induce the plasma, acquire the spectra, and automatically discriminate or detect materials is feasible. It could result in an essential instrument in many real applications, like the ones that are being explored today and are described in the following section.

\section{LIBS Applications}

LIBS is useful in a wide range of fields, namely, those which can benefit from a quick chemical analysis at the atomic level, without sample preparation, or even in the field. This paragraph compiles the most important applications at this moment.

5.1. LIBS in Archeology and Cultural Heritage. Samples with archeological or cultural value are sometimes difficult to analyze. These samples cannot usually be moved or destroyed for analysis, and some chemical techniques to prepare the sample or a controlled environment in a laboratory are needed. In the first place, portable LIBS devices can be used, solving the problem when the sample cannot be moved. In the second place, LIBS does not need contact to analyze the sample, avoiding damage in valuable samples. Although LIBS ablates an amount of the sample, the crater is nearly microscopic and practically invisible to the eye. In addition, this microscopic ablated surface improves the spatial resolution, providing accurate spatial analysis and even a depth profile analysis of the sample. The sample does not need to be prepared; hence the analysis is clean and fast. Besides, LIBS probes based on optical fibers allow the analysis of samples with difficult access. Despite these facts, LIBS is a microdestructive technique and the researcher should pay attention to experimental parameters in order to avoid critical damage in valuable samples. Many cultural heritage artifacts can be analyzed with the right LIBS set-up.

LIBS is feasible with virtually all types of materials, for instance ceramics, marble, bones, or metals, usually applying quantitative analysis. The most common analysis attempts to determine the elemental composition of the sample in order to help to date it [74-77], but it works with bones for analysis of paleodiet [75]. LIBS has been used with delicate samples such as Roman coins [78] or other metallic alloys like bronze [74], even under water [79]. In the field of painting, it can determine the elements that compose the pigments. This analysis of pigments can help to date and authenticate frescos or paintings [80]. Moreover, LIBS can be used, combined with other techniques, in order to sum the potential of them, such as Raman or X-ray fluorescence (XRF) [78, 81, 82].

5.2. LIBS in Biomedical Applications. Biomedicine and LIBS are fields that have not been working together for long. For that reason, this field may provide a large number of new developments in a few years. LIBS can analyze chemical compositions of biological samples such as human bones, tissues, and fluids [83].

LIBS can help to detect excess or deficiency of minerals in tissue, teeth, nails, or bones, as well as toxic elements [83, 84]. In the same way, cancer detection is possible with LIBS and it can provide a surgical device which can detect and destroy tumor cells at the same time [85]. In addition, classification of pathogenic bacteria or virus is possible too $[86,87]$.

The analysis of samples from plants is difficult, because they need a difficult preparation of the sample based on acid digestion processes in order to obtain accurate analysis of micronutrients. LIBS can provide a fast analysis tool with easy sample preparation, for instance in micronutrient analysis of leaves [88].

5.3. LIBS in Industry. LIBS has been targeting many industrial processes for many years, because it is a fast analytical tool well suited to controlling some manufacturing process. Moreover, LIBS can work at a large range of distances, allowing analysis of samples in hazardous and harsh environments. For example, remote detection of explosives has been assessed with LIBS [89], even at trace levels [90].

In the nuclear energy industry, the effects of radiation on living beings and devices are widely known. LIBS can work far away from nuclear waste or reactors, using stand-off configuration or with fiber optic probes, avoiding dangerous radiation levels $[91,92]$.

In the metallurgical industry, smelters, and final products can reach high temperatures, and LIBS can analyze the alloy compositions in production line or detect impurities in other production sectors, such as the automotive industries [71, 93-95].

LIBS can also be useful to detect toxic products like heavy metals in industrial wastes [96]. These waste products should be recycled or stored, and knowing the elements in them can provide key data to reduce the environmental impact of the process.

In the renewable energy field, analysis and detection of impurities in solar cells can be a useful tool to improve the manufacturing processes or to achieve high efficiency solar panels. There are recent research works in this field [97] although there is a huge amount of work to do. 
5.4. LIBS and Geological Samples: Towards Extraterrestrial Limits. Analysis of some kinds of minerals is possible using LIBS, in particular, of soils and geological samples in situ [98]. Sample features can strongly affect the experimental conditions and reduce the accuracy, but quantitative analysis is still possible [99]. LIBS analysis can detect traces of toxic material in soils, rocks, or water without sample preparation and in the natural environment of the sample.

LIBS can work in a wide range of environmental conditions and with different atmospheres, from air to vacuum. This feature, coupled with the capability to analyze soil samples and the possibility to build a portable set-up, enables the possibility to work in the space. Recently, a spacecraft has been launched to Mars to provide spectral analysis of Mars, geological samples $[58,100]$. This spacecraft contains, among other things, a hybrid LIBS-Raman spectrometer.

\section{LIBS Challenges}

Probably, the main challenge that LIBS needs to address is its recognition as a standard in chemical quantitative analysis. Calibration-free algorithms offer a good approximation to this goal, but the results are not perfect yet [101]. There are different research lines with the goal of a standard quantitative analysis, attempting to improve the calibrationfree algorithm or add new capabilities to it. There are recent works based on spectral normalization to improve the final result [101] or to detect the elements in the sample automatically [102]. This goal may be the most important and could place LIBS definitively among the most widely used spectrochemical techniques.

In order to widen its use in real applications, new advanced and cost-effective instrumentation is required. Currently, a cumbersome and expensive set-up is needed to achieve accurate analysis, and work is in progress to reduce the size and complexity of LIBS set-ups. New work in microLIBS and improvements in laser sources useful to LIBS can enable a compact and accurate set-up which makes it feasible in field work [101]. A recent ("hyphenated") approach combines LIBS with other spectrochemical techniques in order to unite the features of them. The Mars Science Laboratory (MSL) is a good example of this because it is a hybrid LIBS-Raman system [58, 100].

Advances in new techniques and approaches for LIBS analysis, such as optical catapulting and molecular LIBS are being explored. Optical catapulting LIBS (OC-LIBS) $[103,104]$ uses a pulsed laser below the plasma threshold energy on the sample surface to create a solid aerosol which is analyzed with LIBS. Molecular LIBS, on the other hand, analyzes the emission of molecules resulting from sample ablation or from the recombination between target elements and ambient air [101]. LIBS can improve its performance with this ability and so enable the analysis of organic samples [105].

\section{Conclusions}

LIBS is a useful spectrochemical technique that can provide chemical analysis in situ without sample preparation, in a quasinondestructive way, which can be used in a wide range of environments.

Nevertheless, advances in key optical components, preprocessing, and postprocessing algorithms in LIBS are essential to go beyond the spectral signatures. Therefore, additional research effort is required to meet the aforementioned challenges and to obtain useful, cost-effective, portable LIBS instrumentation.

\section{Acknowledgment}

This work has been cosupported by the Spanish Government through the Project TEC2010-20224-C02-C02 and Grant AP2007-02230.

\section{References}

[1] B. Kearton and Y. Mattley, "Laser-induced breakdown spectroscopy: sparking new applications," Nature Photonics, vol. 2, no. 9, pp. 537-540, 2008.

[2] D. A. Cremers, L. J. Radziemski, and J. Wiley, Handbook of Laser-Induced Breakdown Spectroscopy, John Wiley \& Sons, 2006.

[3] A. W. Miziolek, V. Palleschi, and I. Schechter, Laser-Induced Breakdown Spectroscopy (LIBS): Fundamentals and Applications, Cambridge University Press, 2006.

[4] W. B. Lee, J. Wu, Y. I. Lee, and J. Sneddon, "Recent applications of laser-induced breakdown spectrometry: a review of material approaches," Applied Spectroscopy Reviews, vol. 39, no. 1, pp. 27-97, 2004.

[5] J. D. Winefordner, I. B. Gornushkin, T. Correll, E. Gibb, B. W. Smith, and N. Omenetto, "Comparing several atomic spectrometric methods to the super stars: special emphasis on laser induced breakdown spectrometry, LIBS, a future super star," Journal of Analytical Atomic Spectrometry, vol. 19, no. 9, pp. 1061-1083, 2004.

[6] B. Cagnac and J. C. Pebay-Peyroula, Modern Atomic Physics: Fundamental Principles, John Wiley \& Sons, 1975.

[7] J. P. Singh, Laser-Induced Breakdown Spectroscopy, Elsevier Science, 2007.

[8] D. Bulajic, M. Corsi, G. Cristoforetti et al., "A procedure for correcting self-absorption in calibration free-laser induced breakdown spectroscopy," Spectrochimica Acta Part B, vol. 57, no. 2, pp. 339-353, 2002.

[9] M. Corsi, G. Cristoforetti, M. Hidalgo et al., "Double pulse, calibration-free laser-induced breakdown spectroscopy: a new technique for in situ standard-less analysis of polluted soils," Applied Geochemistry, vol. 21, no. 5, pp. 748-755, 2006.

[10] H. R. Griem, Spectral Line Broadening by Plasmas, vol. 39 of Pure and Applied Physics, Academic Press, New York, NY, USA, 1974.

[11] G. Cristoforetti, A. De Giacomo, M. Dell'Aglio et al., "Local thermodynamic equilibrium in laser-induced breakdown spectroscopy: beyond the McWhirter criterion," Spectrochimica Acta Part B, vol. 65, no. 1, pp. 86-95, 2010.

[12] J. M. Vadillo, J. M. Fernández Romero, C. Rodríguez, and J. J. Laserna, "Effect of plasma shielding on laser ablation rate of pure metals at reduced pressure," Surface and Interface Analysis, vol. 27, no. 11, pp. 1009-1015, 1999.

[13] J. A. Aguilera, C. Aragón, and F. Peñalba, "Plasma shielding effect in laser ablation of metallic samples and its influence 
on LIBS analysis," Applied Surface Science, vol. 127-129, pp. 309-314, 1998.

[14] S. M. Angel, D. N. Stratis, K. L. Eland, T. Lai, M. A. Berg, and D. M. Gold, "LIBS using dual- and ultra-short laser pulses," Fresenius' Journal of Analytical Chemistry, vol. 369, no. 1, pp. 320-327, 2001.

[15] Y. B. Zel'Dovich and Y. P. Raizer, Physics of Shock Waves and High-Temperature Hydrodynamic Phenomena, Dover, 2002.

[16] L. Sedov, Similarity Methods and Dimensional Analysis in Mechanics, Izdatel Nauka, Moscow, Russia, 1977.

[17] B. Le Drogoff, J. Margot, M. Chaker et al., "Temporal characterization of femtosecond laser pulses induced plasma for spectrochemical analysis of aluminum alloys," Spectrochimica Acta Part B, vol. 56, no. 6, pp. 987-1002, 2001.

[18] X. Zeng, X. L. Mao, R. Greif, and R. E. Russo, "Experimental investigation of ablation efficiency and plasma expansion during femtosecond and nanosecond laser ablation of silicon," Applied Physics A, vol. 80, no. 2, pp. 237-241, 2005.

[19] A. Semerok and C. Dutouquet, "Ultrashort double pulse laser ablation of metals," Thin Solid Films, vol. 453-454, pp. 501505, 2004.

[20] B. C. Castle, K. Talabardon, B. W. Smith, and J. D. Winefordner, "Variables influencing the precision of laserinduced breakdown spectroscopy measurements," Applied Spectroscopy, vol. 52, no. 5, pp. 649-657, 1998.

[21] Y. Iida, "Effects of atmosphere on laser vaporization and excitation processes of solid samples," Spectrochimica Acta Part B, vol. 45, no. 12, pp. 1353-1367, 1990.

[22] C. López-Moreno, S. Palanco, J. J. Laserna et al., “Test of a stand-off laser-induced breakdown spectroscopy sensor for the detection of explosive residues on solid surfaces," Journal of Analytical Atomic Spectrometry, vol. 21, no. 1, pp. 55-60, 2006.

[23] J. L. Gottfried, F. C. De Lucia, C. A. Munson, and A. W. Miziolek, "Double-pulse standoff laser-induced breakdown spectroscopy for versatile hazardous materials detection," Spectrochimica Acta Part B, vol. 62, no. 12, pp. 1405-1411, 2007.

[24] L. St-Onge, M. Sabsabi, and P. Cielo, "Analysis of solids using laser-induced plasma spectroscopy in double-pulse mode," Spectrochimica Acta Part B, vol. 53, no. 2-14, pp. 407-415, 1998.

[25] G. Abdellatif and H. Imam, "A study of the laser plasma parameters at different laser wavelengths," Spectrochimica Acta Part B, vol. 57, no. 7, pp. 1155-1165, 2002.

[26] L. M. Cabalin and J. J. Laserna, "Experimental determination of laser induced breakdown thresholds of metals under nanosecond Q-switched laser operation," Spectrochimica Acta Part B, vol. 53, no. 5, pp. 723-730, 1998.

[27] R. E. Russo, X. L. Mao, O. V. Borisov, and L. Haichen, "Influence of wavelength on fractionation in laser ablation ICP-MS," Journal of Analytical Atomic Spectrometry, vol. 15, no. 9, pp. 1115-1120, 2000.

[28] X. Mao, W. T. Chan, M. Caetano, M. A. Shannon, and R. E. Russo, "Preferential vaporization and plasma shielding during nano-second laser ablation," Applied Surface Science, vol. 96-98, pp. 126-130, 1996.

[29] W. Koechner, Solid-State Laser Engineering, Springer, 2006.

[30] X. L. Mao, A. C. Ciocan, O. V. Borisov, and R. E. Russo, "Laser ablation processes investigated using inductively coupled plasma-atomic emission spectroscopy (ICP-AES)," Applied Surface Science, vol. 127-129, pp. 262-268, 1998.

[31] A. Ciucci, V. Palleschi, S. Rastelli et al., "Trace pollutants analysis in soil by a time-resolved laser-induced breakdown spectroscopy technique," Applied Physics B, vol. 63, no. 2, pp. 185-190, 1997.

[32] R. Wisbrun, I. Schechter, R. Niessner, H. Schröder, and K. L. Kompa, "Detector for trace elemental analysis of solid environmental samples by laser plasma spectroscopy," Analytical Chemistry, vol. 66, no. 18, pp. 2964-2975, 1994.

[33] D. A. Cremers, L. J. Radziemski, and T. R. Loree, "Spectrochemical analysis of liquids using the laser spark," Applied Spectroscopy, vol. 38, no. 5, pp. 721-729, 1984.

[34] R. Sattmann, V. Sturm, and R. Noll, "Laser-induced breakdown spectroscopy of steel samples using multiple Q-switch Nd: YAG laser pulses," Journal of Physics D, vol. 28, article 2181, 1995.

[35] D. N. Stratis, K. L. Eland, and S. M. Angel, "Dual-pulse LIBS using a pre-ablation spark for enhanced ablation and emission," Applied Spectroscopy, vol. 54, no. 9, pp. 1270-1274, 2000.

[36] J. Uebbing, J. Brust, W. Sdorra, F. Leis, and K. Niemax, "Reheating of a laser-produced plasma by a second pulse laser," Applied Spectroscopy, vol. 45, no. 9, pp. 1419-1423, 1991.

[37] D. N. Stratis, K. L. Eland, and S. M. Angel, "Effect of pulse delay time on a pre-ablation dual-pulse LIBS plasma," Applied Spectroscopy, vol. 55, no. 10, pp. 1297-1303, 2001.

[38] D. N. Stratis, K. L. Eland, and S. M. Angel, "Enhancement of aluminum, titanium, and iron in glass using pre-ablation spark dual-pulse LIBS," Applied Spectroscopy, vol. 54, no. 12, pp. 1719-1726, 2000.

[39] J. Scaffidi, W. Pearman, J. C. Carter, B. W. Colston, and S. M. Angel, "Temporal dependence of the enhancement of material removal in femtosecond-nanosecond dual-pulse laser-induced breakdown spectroscopy," Applied Optics, vol. 43, no. 35, pp. 6492-6499, 2004.

[40] J. F. James and R. Sternberg, The Design of Optical Spectrometers, Chapman \& Hall, London, UK, 1969.

[41] H. E. Bauer, F. Leis, and K. Niemax, "Laser induced breakdown spectrometry with an echelle spectrometer and intensified charge coupled device detection," Spectrochimica Acta Part B, vol. 53, no. 13, pp. 1815-1825, 1998.

[42] J. E. Carranza, E. Gibb, B. W. Smith, D. W. Hahn, and J. D. Winefordner, "Comparison of nonintensified and intensified CCD detectors for laser-induced breakdown spectroscopy," Applied Optics, vol. 42, no. 30, pp. 6016-6021, 2003.

[43] G. A. Theriault, S. Bodensteiner, and S. H. Lieberman, "A Real-Time Fiber-Optic LIBS Probe for the in Situ Delineation of Metals in Soils," Field Analytical Chemistry and Technology, vol. 2, no. 2, pp. 117-125, 1998.

[44] B. J. Marquardt, S. R. Goode, and S. Michael Angel, "In situ determination of lead in paint by laser-induced breakdown spectroscopy using a fiber-optic probe," Analytical Chemistry, vol. 68, no. 6, pp. 977-981, 1996.

[45] A. K. Rai, H. Zhang, Fang Yu Yueh, J. P. Singh, and A. Weisburg, "Parametric study of a fiber-optic laser-induced breakdown spectroscopy probe for analysis of aluminum alloys," Spectrochimica Acta Part B, vol. 56, no. 12, pp. 23712383, 2001.

[46] D. Cremers, J. Barefield, and A. Koskelo, "Remote elemental analysis by laser-induced breakdown spectroscopy using a fiber-optic cable," Applied Spectroscopy, vol. 49, pp. 857-860, 1995.

[47] S. O. Konorov, A. B. Fedotov, O. A. Kolelvlatova et al., "Laser breakdown with millijoule trains of picosecond pulse transmitted through a hollow-core photonic-crystal fibre," Journal of Physics D, vol. 36, no. 12, pp. 1375-1381, 2003. 
[48] L. Rodríguez-Cobo, F. Anabitarte, M. Lomer, J. Mirapeix, J. M. Lopez-Higuera, and A. Cobo, "Laser Induced Breakdown Spectroscopy light collector based on coiled plastic optical fiber," in International conference on Plastic Optical fibers POF, Bilbao, Spain, 2011.

[49] M. A. Losada, I. Garcés, J. Mateo, I. Salinas, J. Lou, and J. Zubía, "Mode coupling contribution to radiation losses in curvatures for high and low numerical aperture plastic optical fibers," Journal of Lightwave Technology, vol. 20, no. 7, pp. 1160-1164, 2002.

[50] J. Laserna and S. Palanco, "Spectral analysis of the acoustic emission of laser-produced plasmas," Applied Optics, vol. 42, no. 30, pp. 6078-6084, 2003.

[51] A. Hrdlička, L. Zaorálková, M. Galiová et al., "Correlation of acoustic and optical emission signals produced at 1064 and $532 \mathrm{~nm}$ laser-induced breakdown spectroscopy (LIBS) of glazed wall tiles," Spectrochimica Acta Part B, vol. 64, no. 1, pp. 74-78, 2009.

[52] K. O. Hill and G. Meltz, "Fiber Bragg grating technology fundamentals and overview," Journal of Lightwave Technology, vol. 15, no. 8, pp. 1263-1276, 1997.

[53] J. M. López-Higuera, L. R. Cobo, A. Q. Incera, and A. Cobo, "Fiber optic sensors in structural health monitoring," Journal of Lightwave Technology, vol. 29, no. 4, pp. 587-608, 2011.

[54] F. Anabitarte, L. Rodríguez-Cobo, J. Mirapeix, J. M. LópezHiguera, and A. Cobo, "Acoustic Detection of laser-induced plasma emission by means of a fiber-Bragg grating sensor," in Proceedings of the 7th Reunion Española de Optoelectronica OPTOEL, Santander, Spain, 2011.

[55] Y. Ralchenko, "NIST atomic spectra database," Memorie Della Societa Astronomica Italiana Supplementi, vol. 8, p. 96, 2005.

[56] I. Bassiotis, A. Diamantopoulou, A. Giannoudakos, F. Roubani-Kalantzopoulou, and M. Kompitsas, "Effects of experimental parameters in quantitative analysis of steel alloy by laser-induced breakdown spectroscopy," Spectrochimica Acta Part B, vol. 56, no. 6, pp. 671-683, 2001.

[57] R. A. Multari, L. E. Foster, D. A. Cremers, and M. J. Ferris, "Effect of sampling geometry on elemental emissions in laser-induced breakdown spectroscopy," Applied Spectroscopy, vol. 50, no. 12, pp. 1483-1499, 1996.

[58] B. Sallé, D. A. Cremers, S. Maurice, and R. C. Wiens, "Laser-induced breakdown spectroscopy for space exploration applications: influence of the ambient pressure on the calibration curves prepared from soil and clay samples," Spectrochimica Acta Part B, vol. 60, no. 4, pp. 479-490, 2005.

[59] A. S. Eppler, D. A. Cremers, D. D. Hickmott, M. J. Ferris, and A. C. Koskelo, "Matrix effects in the detection of $\mathrm{Pb}$ and $\mathrm{Ba}$ in soils using laser-induced breakdown spectroscopy," Applied Spectroscopy, vol. 50, no. 9, pp. 1175-1181, 1996.

[60] L. St-Onge, E. Kwong, M. Sabsabi, and E. B. Vadas, "Quantitative analysis of pharmaceutical products by laser-induced breakdown spectroscopy," Spectrochimica Acta Part B, vol. 57, no. 7, pp. 1131-1140, 2002.

[61] C. Chaléard, P. Mauchien, N. Andre, J. Uebbing, J. L. Lacour, and C. Geertsen, "Correction of matrix effects in quantitative elemental analysis with laser ablation optical emission spectrometry," Journal of Analytical Atomic Spectrometry, vol. 12, no. 2, pp. 183-188, 1997.

[62] A. Ciucci, M. Corsi, V. Palleschi, S. Rastelli, A. Salvetti, and E. Tognoni, "New procedure for quantitative elemental analysis by laser-induced plasma spectroscopy," Applied Spectroscopy, vol. 53, no. 8, pp. 960-964, 1999.

[63] B. Yegnanarayana, Artificial Neural Networks, PHI Learning Pvt. Ltd., 2004.
[64] V. N. Vapnik, The Nature of Statistical Learning Theory, Springer, 2000.

[65] C. Cortes and V. Vapnik, "Support-vector networks," Machine Learning, vol. 20, no. 3, pp. 273-297, 1995.

[66] N. Roussopoulos, S. Kelley, and F. Vincent, "Nearest neighbor queries," in Proceedings of the ACM SIGMOD International Conference on Management of Data, pp. 71-79, 1995.

[67] J. M. Keller, M. R. Gray, and J. A. Givens, "A fuzzy K-nearestneighbor algorithm," IEEE Transactions on Systems, Man and Cybernetics, vol. 15, no. 4, article 581, 1985.

[68] P. Pudil, J. Novovičová, and J. Kittler, "Floating search methods in feature selection," Pattern Recognition Letters, vol. 15, no. 11, pp. 1119-1125, 1994.

[69] H. Yu and J. Yang, "A direct LDA algorithm for highdimensional data-with application to face recognition," Pattern Recognition, vol. 34, pp. 2067-2070, 2001.

[70] S. Wold, K. Esbensen, and P. Geladi, "Principal component analysis," Chemometrics and Intelligent Laboratory Systems, vol. 2, no. 1-3, pp. 37-52, 1987.

[71] F. Anabitarte, J. Mirapeix, O. M. C. Portilla, J. M. LopezHiguera, and A. Cobo, "Sensor for the detection of protective coating traces on boron steel with aluminium-silicon covering by means of laser-induced breakdown spectroscopy and support vector machines," IEEE Sensors Journal, vol. 12, no. 1, Article ID Article number5722011, pp. 64-70, 2012.

[72] J. B. Sirven, B. Bousquet, L. Canioni et al., "Qualitative and quantitative investigation of chromium-polluted soils by laser-induced breakdown spectroscopy combined with neural networks analysis," Analytical and Bioanalytical Chemistry, vol. 385, no. 2, pp. 256-262, 2006.

[73] Q. Godoi, F. O. Leme, L. C. Trevizan et al., "Laser-induced breakdown spectroscopy and chemometrics for classification of toys relying on toxic elements," Spectrochimica Acta Part B, vol. 66, no. 2, pp. 138-143, 2011.

[74] L. Fornarini, F. Colao, R. Fantoni, V. Lazic, and V. Spizzicchino, "Calibration analysis of bronze samples by nanosecond laser induced breakdown spectroscopy: a theoretical and experimental approach," Spectrochimica Acta Part B, vol. 60, no. 7-8, pp. 1186-1201, 2005.

[75] M. A. Kasem, R. E. Russo, and M. A. Harith, "Influence of biological degradation and environmental effects on the interpretation of archeological bone samples with laserinduced breakdown spectroscopy," Journal of Analytical Atomic Spectrometry, vol. 26, no. 9, pp. 1733-1739, 2011.

[76] K. Melessanaki, M. Mateo, S. C. Ferrence, P. P. Betancourt, and D. Anglos, "The application of LIBS for the analysis of archaeological ceramic and metal artifacts," Applied Surface Science, vol. 197-198, pp. 156-163, 2002.

[77] P. V. Maravelaki, V. Zafiropulos, V. Kilikoglou, M. Kalaitzaki, and C. Fotakis, "Laser-induced breakdown spectroscopy as a diagnostic technique for the laser cleaning of marble," Spectrochimica Acta Part B, vol. 52, no. 1, pp. 41-53, 1997.

[78] L. Torrisi, F. Caridi, L. Giuffrida et al., "LAMQS analysis applied to ancient Egyptian bronze coins," Nuclear Instruments and Methods in Physics Research, Section B, vol. 268, no. 10, pp. 1657-1664, 2010.

[79] V. Lazic, F. Colao, R. Fantoni, and V. Spizzicchino, "Recognition of archeological materials underwater by laser induced breakdown spectroscopy," Spectrochimica Acta Part B, vol. 60, no. 7-8, pp. 1014-1024, 2005.

[80] L. Caneve, A. Diamanti, F. Grimaldi, G. Palleschi, V. Spizzichino, and F. Valentini, "Analysis of fresco by laser induced breakdown spectroscopy," Spectrochimica Acta Part $B$, vol. 65, no. 8, pp. 702-706, 2010. 
[81] I. Osticioli, N. F. C. Mendes, S. Porcinai, A. Cagnini, and E. Castellucci, "Spectroscopic analysis of works of art using a single LIBS and pulsed Raman setup," Analytical and Bioanalytical Chemistry, vol. 394, no. 4, pp. 1033-1041, 2009.

[82] M. F. Alberghina, R. Barraco, M. Brai, T. Schillaci, and L. Tranchina, "Comparison of LIBS and $\mu$-XRF measurements on bronze alloys for monitoring plasma effects," Journal of Physics, vol. 275, no. 1, Article ID 012017, 2011.

[83] X. Y. Liu and W. J. Zhang, "Recent developments in biomedicine fields for laser induced breakdown spectroscopy," Journal of Biomedical Science, vol. 1, pp. 147-151, 2008.

[84] S. Hamzaoui, R. Khleifia, N. Jaïdane, and Z. Ben Lakhdar, "Quantitative analysis of pathological nails using laserinduced breakdown spectroscopy (LIBS) technique," Lasers in Medical Science, vol. 26, no. 1, pp. 79-83, 2011.

[85] C. Tameze, R. Vincelette, N. Melikechi, V. Zeljković, and E. Izquierdo, "Empiricalanalysis of LIBS images for ovarian cancer detection," in Proceedings of the 8th International Workshop on Image Analysis for Multimedia Interactive Services (WIAMIS '07), p. 76, June 2007.

[86] S. J. Rehse, Q. I. Mohaidat, and S. Palchaudhuri, “Towards the clinical application of laser-induced breakdown spectroscopy for rapid pathogen diagnosis: the effect of mixed cultures and sample dilution on bacterial identification," Applied Optics, vol. 49, no. 13, pp. C27-C35, 2010.

[87] R. A. Multari, D. A. Cremers, and M. L. Bostian, "Use of laserinduced breakdown spectroscopy for the differentiation of pathogens and viruses on substrates," Applied Optics, vol. 51, no. 7, pp. B57-B64, 2012.

[88] L. C. Trevizan, D. Santos, R. E. Samad et al., "Evaluation of laser induced breakdown spectroscopy for the determination of micronutrients in plant materials," Spectrochimica Acta Part B, vol. 64, no. 5, pp. 369-377, 2009.

[89] R. González, P. Lucena, L. M. Tobaria, and J. J. Laserna, "Standoff LIBS detection of explosive residues behind a barrier," Journal of Analytical Atomic Spectrometry, vol. 24, no. 8, pp. 1123-1126, 2009.

[90] V. Lazic, A. Palucci, S. Jovicevic, M. Carapanese, C. Poggi, and E. Buono, "Detection of explosives at trace levels by Laser Induced Breakdown Spectroscopy (LIBS)," in Chemical, Biological, Radiological, Nuclear, and Explosives (CBRNE) Sensing XI, vol. 7665 of Proceedings of SPIE, April 2010.

[91] A. I. Whitehouse, J. Young, I. M. Botheroyd, S. Lawson, C. P. Evans, and J. Wright, "Remote material analysis of nuclear power station steam generator tubes by laser-induced breakdown spectroscopy," Spectrochimica Acta Part B, vol. 56, no. 6, pp. 821-830, 2001.

[92] A. Sarkar, V. M. Telmore, D. Alamelu, and S. K. Aggarwal, "Laser induced breakdown spectroscopic quantification of platinum group metals in simulated high level nuclear waste," Journal of Analytical Atomic Spectrometry, vol. 24, no. 11, pp. 1545-1550, 2009.

[93] Q. J. Guo, H. B. Yu, Y. Xin, X. L. Li, and X. H. Li, "Experimental study on high alloy steel sample by laserinduced breakdown spectroscopy," Guang Pu Xue Yu Guang Pu Fen Xi, vol. 30, no. 3, pp. 783-787, 2010.

[94] C. Aragon, J. Aguilera, and J. Campos, "Determination of carbon content in molten steel using laser-induced breakdown spectroscopy," Applied Spectroscopy, vol. 47, pp. 606608, 1993.

[95] A. K. Rai, F. Y. Yueh, and J. P. Singh, "Laser-induced breakdown spectroscopy of molten aluminum alloy," Applied Optics, vol. 42, no. 12, pp. 2078-2084, 2003.
[96] N. K. Rai and A. K. Rai, "LIBS-An efficient approach for the determination of $\mathrm{Cr}$ in industrial wastewater," Journal of Hazardous Materials, vol. 150, no. 3, pp. 835-838, 2008.

[97] J. M. D. Kowalczyk, J. Perkins, J. Kaneshiro et al., "Measurement of the sodium concentration in CIGS solar cells via laser induced breakdown spectroscopy," in Proceedings of the 35th IEEE Photovoltaic Specialists Conference (PVSC '10), pp. 1742-1744, June 2010.

[98] J. Cuñat, S. Palanco, F. Carrasco, M. D. Simón, and J. J. Laserna, "Portable instrument and analytical method using laser-induced breakdown spectrometry for in situ characterization of speleothems in karstic caves," Journal of Analytical Atomic Spectrometry, vol. 20, no. 4, pp. 295-300, 2005.

[99] J. M. Anzano, M. A. Villoria, A. Ruíz-Medina, and R. J. Lasheras, "Laser-induced breakdown spectroscopy for quantitative spectrochemical analysis of geological materials: effects of the matrix and simultaneous determination," Analytica Chimica Acta, vol. 575, no. 2, pp. 230-235, 2006.

[100] B. Sallé, D. A. Cremers, S. Maurice, R. C. Wiens, and P. Fichet, "Evaluation of a compact spectrograph for in-situ and stand-off Laser-Induced Breakdown Spectroscopy analyses of geological samples on Mars missions," Spectrochimica Acta Part B, vol. 60, no. 6, pp. 805-815, 2005.

[101] D. W. Hahn and N. Omenetto, "Laser-induced breakdown spectroscopy (LIBS), part II: review of instrumental and methodological approaches to material analysis and applications to different fields," Applied Spectroscopy, vol. 66, no. 4, pp. 347-419, 2012.

[102] G. Amato, S. Legnaioli, G. Lorenzetti, V. Palleschi, L. Pardini, and F. Rabitti, "Element detection relying on information retrieval techniques applied to Laser Spectroscopy," in Proceedings of the 4th International Conference on SImilarity Search and APplications (SISAP '11), pp. 89-95, ACM, July 2011.

[103] F. J. Fortes and J. J. Laserna, "Characteristics of solid aerosols produced by optical catapulting studied by laser-induced breakdown spectroscopy," Applied Surface Science, vol. 256, no. 20, pp. 5924-5928, 2010.

[104] M. Abdelhamid, F. J. Fortes, M. A. Harith, and J. J. Laserna, "Analysis of explosive residues in human fingerprints using optical catapulting-laser-induced breakdown spectroscopy," Journal of Analytical Atomic Spectrometry, vol. 26, no. 7, pp. 1445-1450, 2011.

[105] S. Rai and A. K. Rai, "Characterization of organic materials by LIBS for exploration of correlation between molecular and elemental LIBS signals," AIP Advances, vol. 1, no. 4, Article ID 042103, 11 pages, 2011. 


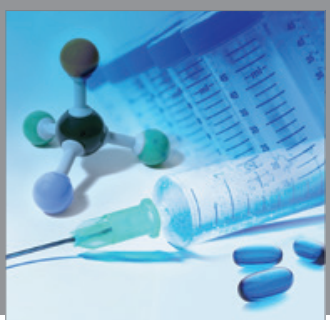

International Journal of

Medicinal Chemistry

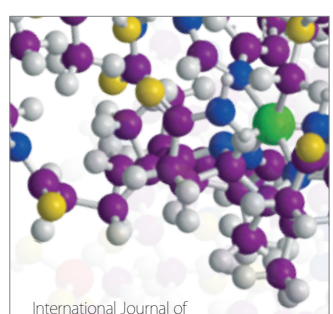

Carbohydrate Chemistry

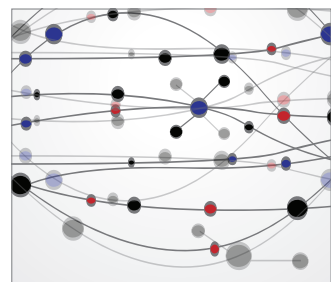

The Scientific World Journal
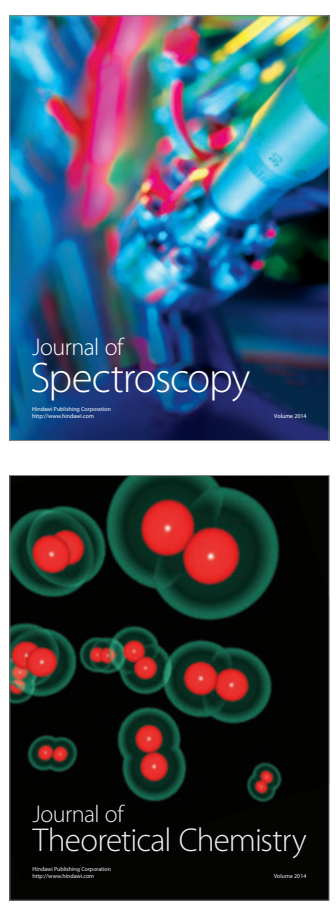
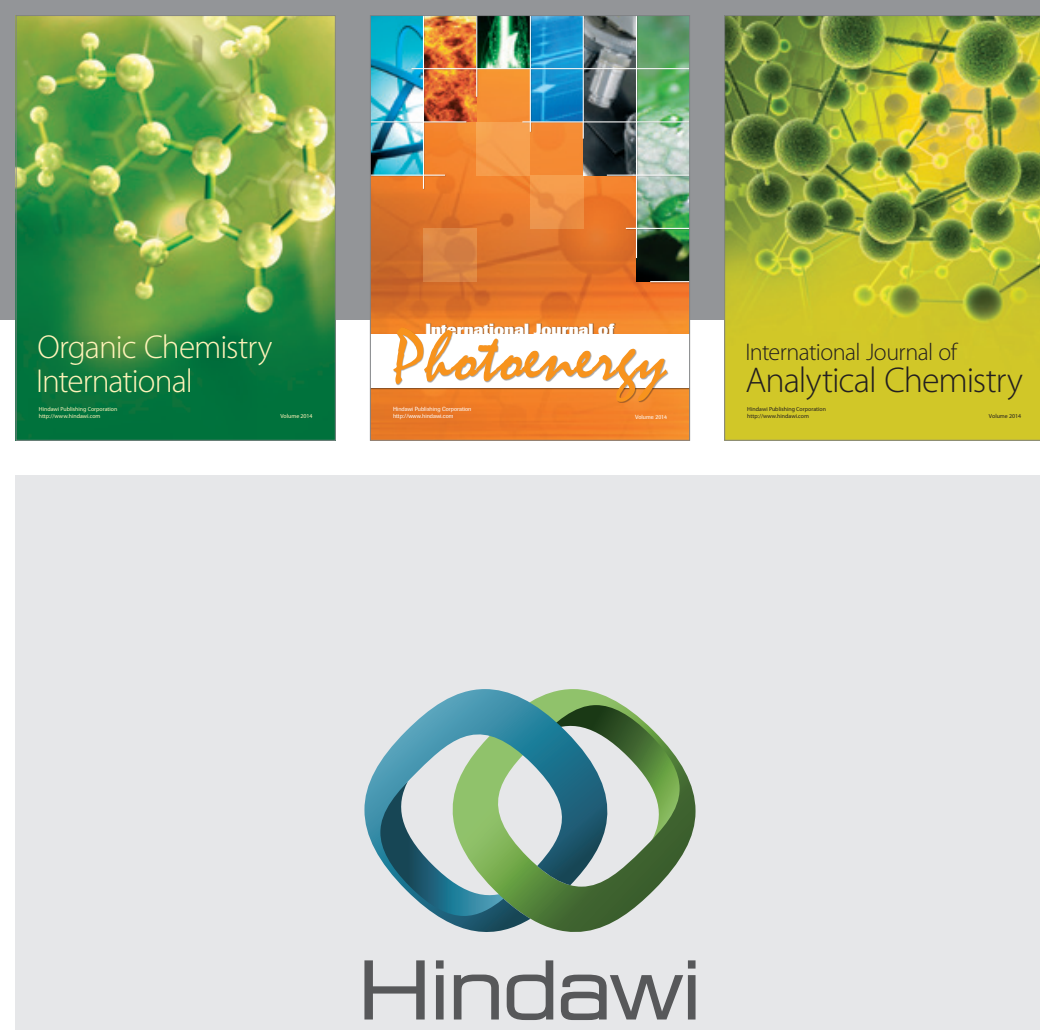

Submit your manuscripts at

http://www.hindawi.com
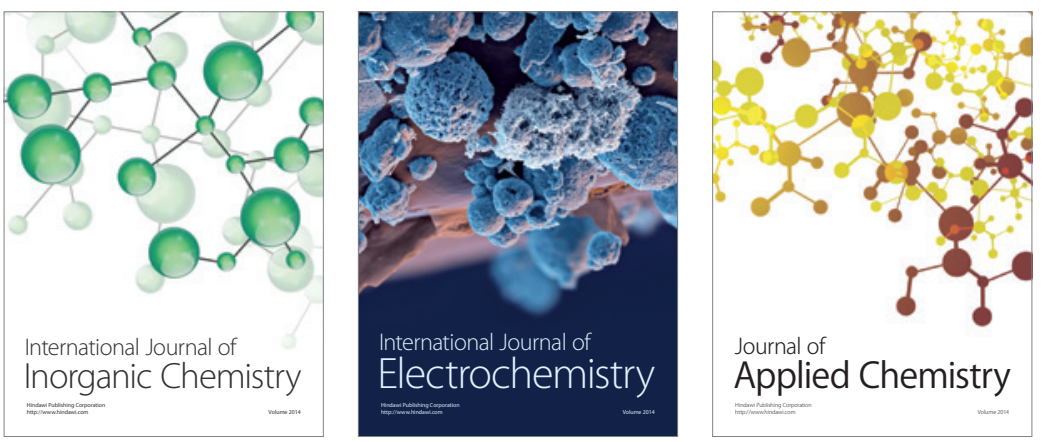

Journal of

Applied Chemistry
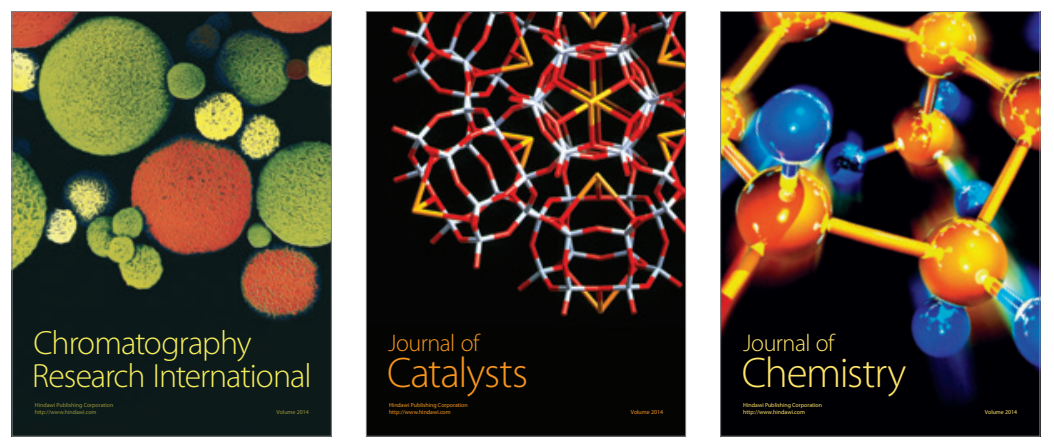
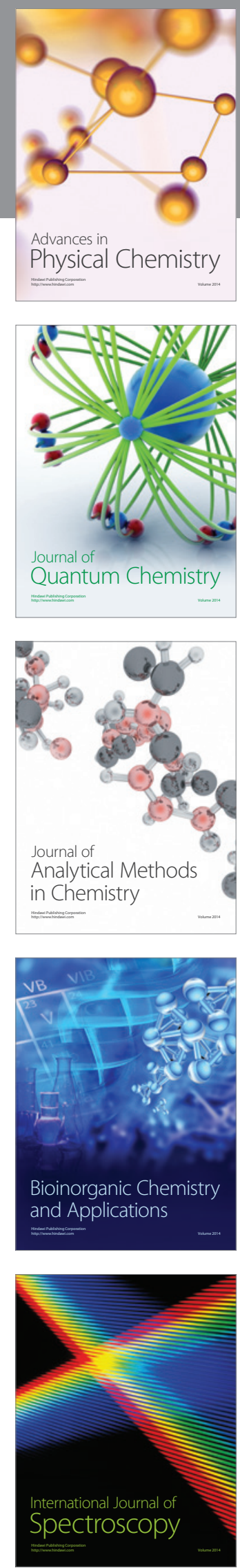